\title{
Research on Diffusion and Control Technology of Welding Fume
}

\author{
Lin Dajian, Zhou Hong, Li Liangfei
}

Department of Resources and Environmental Engineering, Jiangxi University of Science and Technology, Ganzhou, China

Email address:

371138126@qq.com (Lin Dajian),972886058@qq.com (Zhou Hong), 503721636@qq.com (Li Liangfei)

\section{To cite this article:}

Lin Dajian, Zhou Hong, Li Liangfei. Research on Diffusion and Control Technology of Welding Fume. Journal of Energy and Natural Resources. Vol. 6, No. 1, 2017, pp. 1-7. doi: 10.11648/j.jenr.20170601.11

Received: February 14, 2017; Accepted: February 24, 2017; Published: March 18, 2017

\begin{abstract}
Welding fume has the characteristics of small particle size, large damage and difficult to handle. This paper uses the Gambit software and Fluent software to simulate the wind speed, spot spacing and vertical distance factors affecting diffusion welding fume, get the diffusion characteristics of welding fume: in the same section, the greater the spacing, the lower the concentration decreased more quickly; in the same distance, the height of different dust source in the vertical direction, different concentration of welding fumes, smoke concentration is undergoing a process of first increased and then decreased. And put forward, using centrifugal swirl air diffusion, control technology for welding fume entrainment and stratification theory and technology, to achieve effective control of welding fume and energy saving.
\end{abstract}

Keywords: Welding Fume, Spread, Numerical Simulation

\section{Introduction}

Since the 1980s, China has been on the industrialized process, welding work is one of the most important connection way, which is also widely used in industrial production such as metallurgy, machinery, building materials, automobiles, shipbuilding, construction, railway and other industries. In the welding process, the temperature of welding rod and base material is usually higher than $5000^{\circ} \mathrm{C}$, and always produce a large number of welding fume, harmful gases and arc radiation. Dust with particle size between the $0.01 \sim 5 \mu \mathrm{m}$, is mostly the respirable dust. Long-term exposure to high concentrations of dust would cause pneumoconiosis, manganese poisoning, metal fever and other occupational diseases $[1,2]$. The disposition of welding fume is a technical problem, the main difficulties are as follows: 1 it is difficult to filter the small size particle of welding fume, whose size is around $0.01 \sim 5 \mu \mathrm{m}$ diameter. 2 the variability of the welding post makes the welding fume difficult to capture. 3 the hot air retention characteristics of welding fume, 4 high equipment investment and operating costs on the welding fume removing equipment.

There are relatively fewer research in welding fume filed. Marconi and Bravaccini obtain optimum ventilation post for smoke diffusion through the research on the angle of the welding gun and the exhaust rate [3]. CaiZhiPing and $\mathrm{XuZongGu}$ use the theoretical diffusion model to reach the conclusion that the highest concentration distribution of welding fume is obtained in the thermodynamic lifting height [4]. NiuPingPing and Zhao JiangPing analyze the pattern of indoor airflow and the change of soot velocity in different ventilation conditions, and then draw the conclusion that the soot concentration decrease as being closer to the vent, which would even meet the national standards [5]. Though analyzing and testing the distribution of welding fume concentration in different working conditions, combined with national standards on the concentration of welding, Liu ZhiYun and JiaYanYan is studying on the hazards of welding work in different working conditions [6,7]. Though these related research did have a certain positive effect on the study of welding fume, but they do not consider the real diffusion characteristics of welding fume, and they could not collect the welding fume effectively.

The research on the control of the welding fume is mainly to improve the workshop by means of ventilation. LinBao and Dong LiGuo was established an experiment platform for suction-airflow affected by annular jet and designed a ventilation inlet with blades engendering rotating jet [8]; Wang PengFei was established physical model of the rotary air curtain exhaust hood local ventilation system, then using 
Fluent to compute fluid dynamics software for this mode of ventilation flow field numerical simulation, so as to analyze the basic characteristics of the flow field and impact factors include the best angle and the air vents width [9]; Yang Yang and Wang Li thought, when the angle between the jet and the exhaust flow ranges from $90^{\circ}$ to $60^{\circ}$, utilizing the numerical simulation, the conditions to form steady flow was determined and the changes of the centerline velocity and control region on the exhaust flow were studied [10].

In this paper, the welding fume diffusion pattern is obtained though the simulation of the diffusion progress, and the welding fume could be collected by use therotary air curtain exhaust hood, which reduce the spread of welding fume effectively.

\section{Model Establishment}

\subsection{Idealized Model}

Typically, the spread of the welding fume is fluid motion, so Fluent Software is usually used to simulate the diffusion of welding fume, in the simulation process, we supposed that the fluid is incompressible, and the influence of temperature on the airflow field could not be considered. Due to the unstable statement and the diffusion of welding fume, an unsteady-state flow process should be considered.

\subsection{Establish Basic Conservation Equations}

The fluid flow is restricted by the physical conservation laws. Basic conservation laws include the law of conservation of mass, the law of conservation of momentum and the law of conservation of energy. If the flow contains a mixture or interaction of different components, the system must follow the law of conservation of constituents. And the system has to follow the turbulent motion equation if the flow is turbulent $[11,12]$.

1) the law of conservation of mass

$$
\frac{\partial \rho}{\partial t}+\frac{\partial(\rho u)}{\partial x}+\frac{\partial(\rho v)}{\partial y}+\frac{\partial(\rho w)}{\partial z}=0
$$

In the equation, $\rho$-Density, $\mathrm{kg} / \mathrm{m}^{3}, t$-Time, $s, u$ -Speed vector, $u, v, w$-The vector component in three dimension, $\mathrm{m} / \mathrm{s}$.

2) the law of conservation of momentum

$$
\frac{\partial\left(p u_{i}\right)}{\partial t}+\frac{\partial}{\partial x_{j}}\left(p u_{i} u_{j}\right)=-\frac{\partial p}{\partial x_{i}}+\frac{\partial \tau_{i j}}{\partial x_{j}}+\rho g_{i}+F_{i}
$$

In the equation, $\tau_{i j}=\left[v\left(\frac{\partial u_{j}}{\partial x_{i}}\right)+\frac{\partial u_{j}}{\partial x_{i}}\right]-\frac{2}{3} v \sigma_{i j} \frac{\partial u_{i}}{\partial x_{i}} \quad p$ - Static pressure, $P a, \tau_{i j}$ —Stress vector, $\rho g_{i}$ - Gravity vector in direction $i$.

$3)$ the law of conservation of energy

$$
\frac{\partial(\rho T)}{\partial t}+\operatorname{div}(\rho u T)=\operatorname{div}\left[\frac{k}{C_{P}} \operatorname{grad} T\right]+S_{T}
$$

In the equation, $C_{P}$-Specific heat capacity, $K$ - Coefficient of heat conduction, $T$-Temperature; $S_{T}$ - The viscous dissipative term, which is the sum of the fluid heat source and those part of thermal energy that mechanical energy change into under the effect of viscosity.

\subsection{Establish the Model of Numerical}

Take a welding workshop as an object of study, build a model by Gambit Software. The model measurements are 80 $\mathrm{m}$ long, $30 \mathrm{~m}$ wide and $8 \mathrm{~m}$ high, there are two doors in the horizontal and vertical sides with the size in $4 \mathrm{~m} \times 5 \mathrm{~m}$. The lateral distribution 10 ventilation windows in $3 \mathrm{~m} \times 1.2 \mathrm{~m}$. Use Fluent Software to simulate the influence on welding fume diffusion in different situations such as the different distance between dust point sources, the different ventilation and the different location of flue exhauster. The model shown in Figure 1.

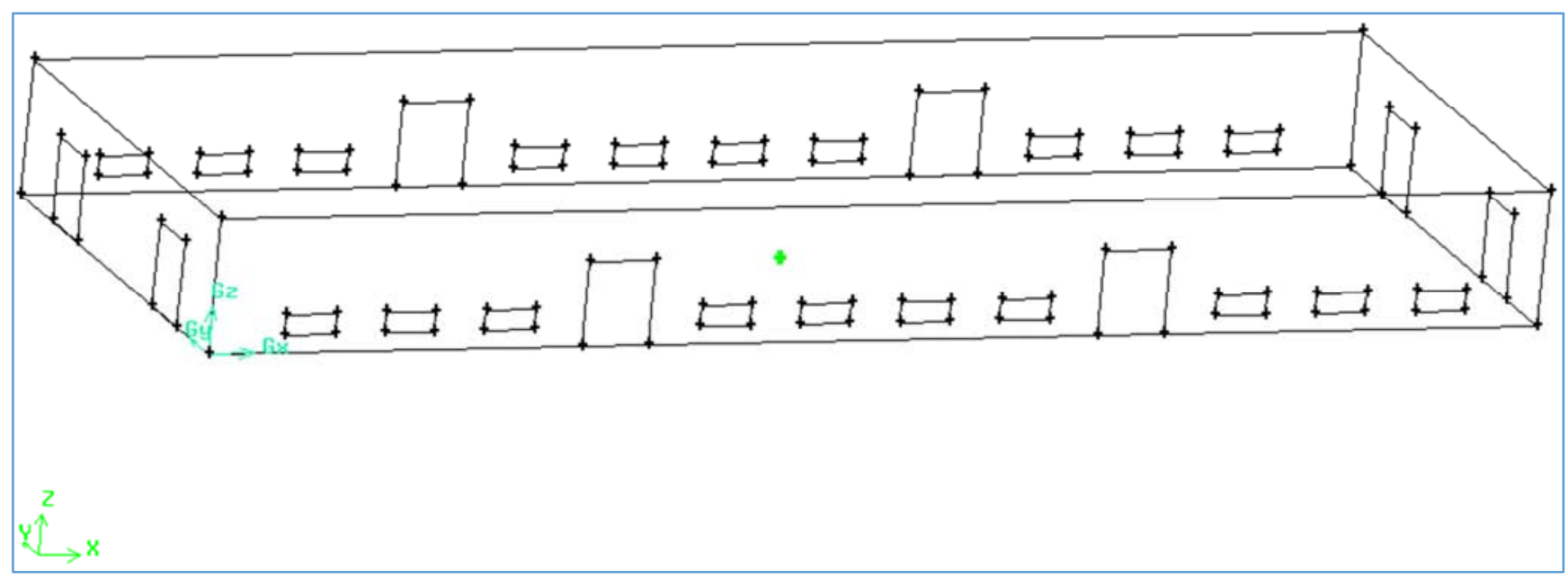

Figure 1. The model of welding workshop. 


\subsection{Determine the Initial and Boundary Condition}

The standard $k-\varepsilon$ turbulence Model is used in figure 1 . Assuming that under the no-wind condition, the workshop is firmly closed, take the dust source in the center of the bottom of the workshop as the mass entrance, and the other part of the workshop could be set as the wall. In the conditions of natural ventilation, take the door and the window as the speed entrance, the other side is free exit.

\subsection{Mesh Division}

The construction of unstructured grid is relatively easy. In addition, when choosing the second-order upwind format, high accuracy is obtained and is more adjustable and controllable. So in this model, the type of unstructured grid is used, and arranged in uniform grid. The term of Elements could choose Tet/Hybrid, and the type could choose TGrid. The divided geometric model shown in Figure 2.

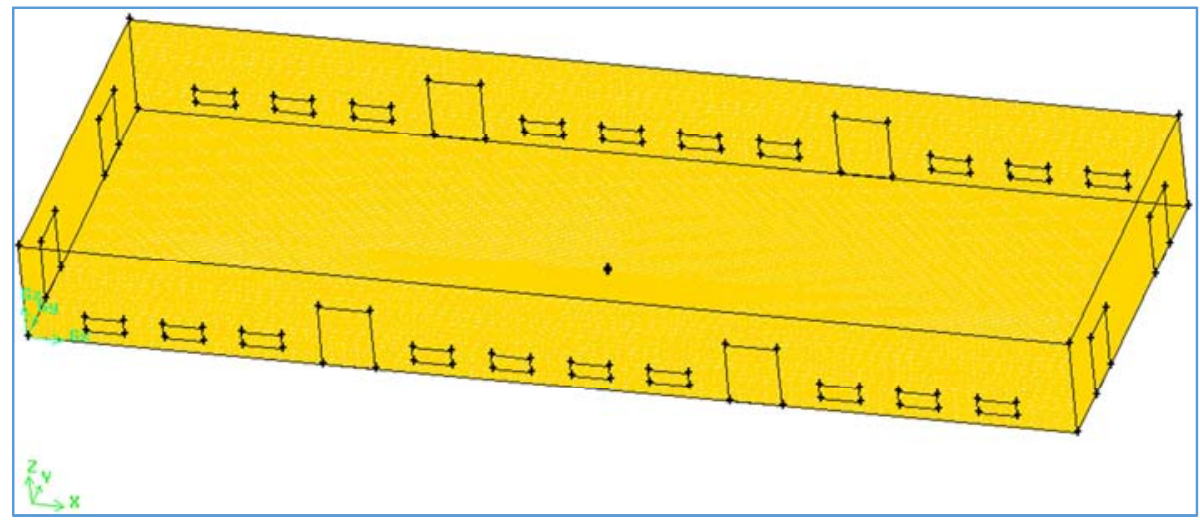

Figure 2. Geometric model after mesh division.

\subsection{Calculate of Control Equation}

Use numerical method to calculate the mathematical model. In Fluent, the finite volume method is used as the discrete method, and using Implicit format, the flow field calculation uses the mode of SIMPLEC pressure-velocity coupling. Use Fluent to set parameters or algorithm then calculate [13].

\section{Simulation and Analysis}

\subsection{Diffusion Simulation of Single Welding Operation}

The following simulation is set under the dust source welding fume diffusion situation and the no-wind condition. The wall and the window in the workshop is represented same as the wall. The diffusion concentration in the center operating point of the workshop bottom whose coordinate is $(40,15,0)$ is shown in Figure 3.

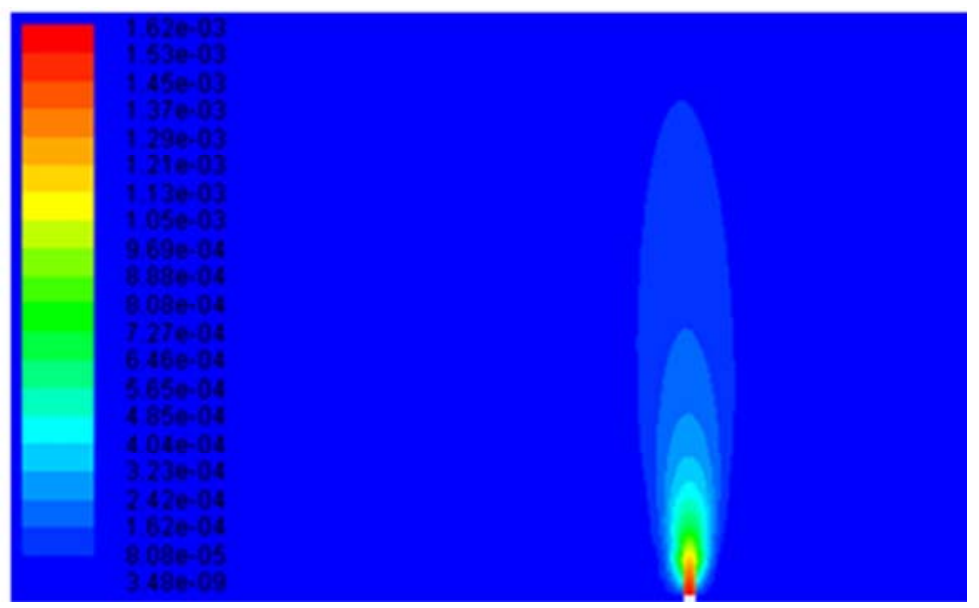

Figure 3. The concentration of welding fume in single operating point.

Keep the $\mathrm{X}$, Y-axis unchangeable, select the $\mathrm{Z}=0.5 \mathrm{~m}, 1 \mathrm{~m}$, $3 \mathrm{~m}$, the condition that dust concentration changed in vertical direction is shown in Figure 4, and keep the X, Z-axis remains, select the $\mathrm{Y}=15 \mathrm{~m}, 14 \mathrm{~m}, 14.7 \mathrm{~m}$, the condition that dust concentration changed in horizontal direction is shown in Figure 5. 


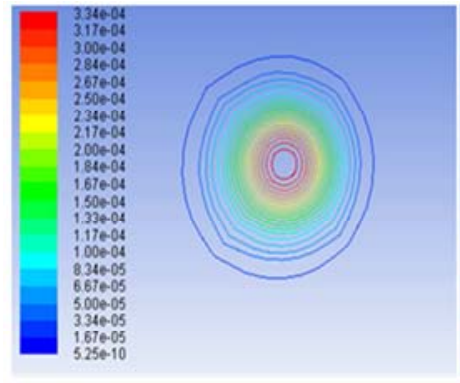

(a) $\mathrm{Z}=0.5 \mathrm{~m}$

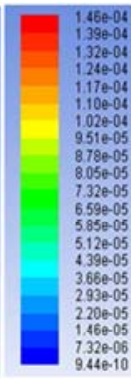

(b) $\mathrm{Z}=1 \mathrm{~m}$

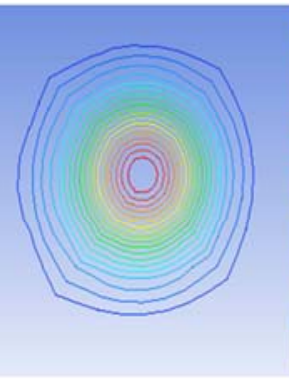

(c) $\mathrm{Z}=3 \mathrm{~m}$

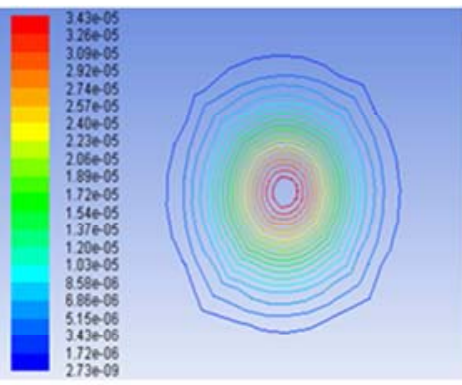

Figure 4. The isoline map of concentration distribution in vertical direction.

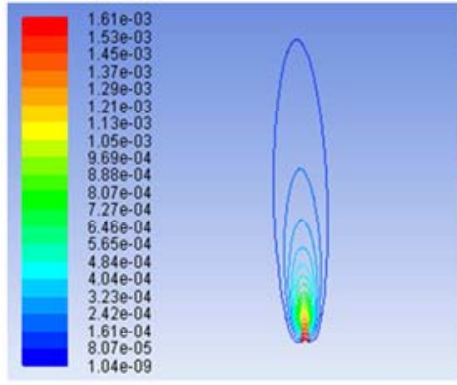

(a) $\mathrm{Y}=15 \mathrm{~m}$

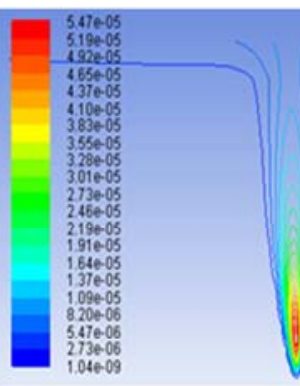

(b) $\mathrm{Y}=14.8 \mathrm{~m}$

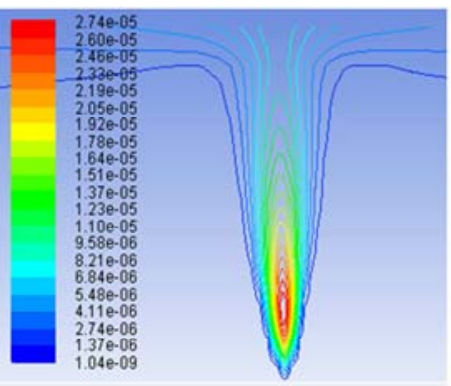

(c) $\quad \mathrm{Y}=14.7 \mathrm{~m}$

Figure 5. The isoline map of concentration distribution in horizontal direction.

As can be seen from Figure 4 and Figure 5, in the no-wind condition, the dust was distributed evenly in vertical direction when keeping the $\mathrm{X}, \mathrm{Y}$-axis unchangeable, and the dust diffusion become more faster as $\mathrm{Z}$ increases. The simulation shows that, when $\mathrm{Z}$ is bigger than $1 \mathrm{~m}$, the concentration of dust diffusion is smaller and the trend is smoother, when keeping the $\mathrm{X}, \mathrm{Z}$-axis unchangeable, dust spread to the surrounding in horizontal direction, and the concentration of dust decreases as the distance increases, this is due to the difference in the density of air formed by the rise of hot air and cold air flow principle [14].

\subsection{Diffusion Simulation of Dense Welding Operation}

\subsubsection{The Influence of the Different Distance of Dust Source on Dust Diffusion}

Set the situation that 9 dust sources exist at the same time under no wind conditions, and the distance between the dust sources is $1.5 \mathrm{~m}, 1.8 \mathrm{~m}, 2.0 \mathrm{~m}$, respectively. The concentration of the welding fume diffusion is shown in Figure 6.

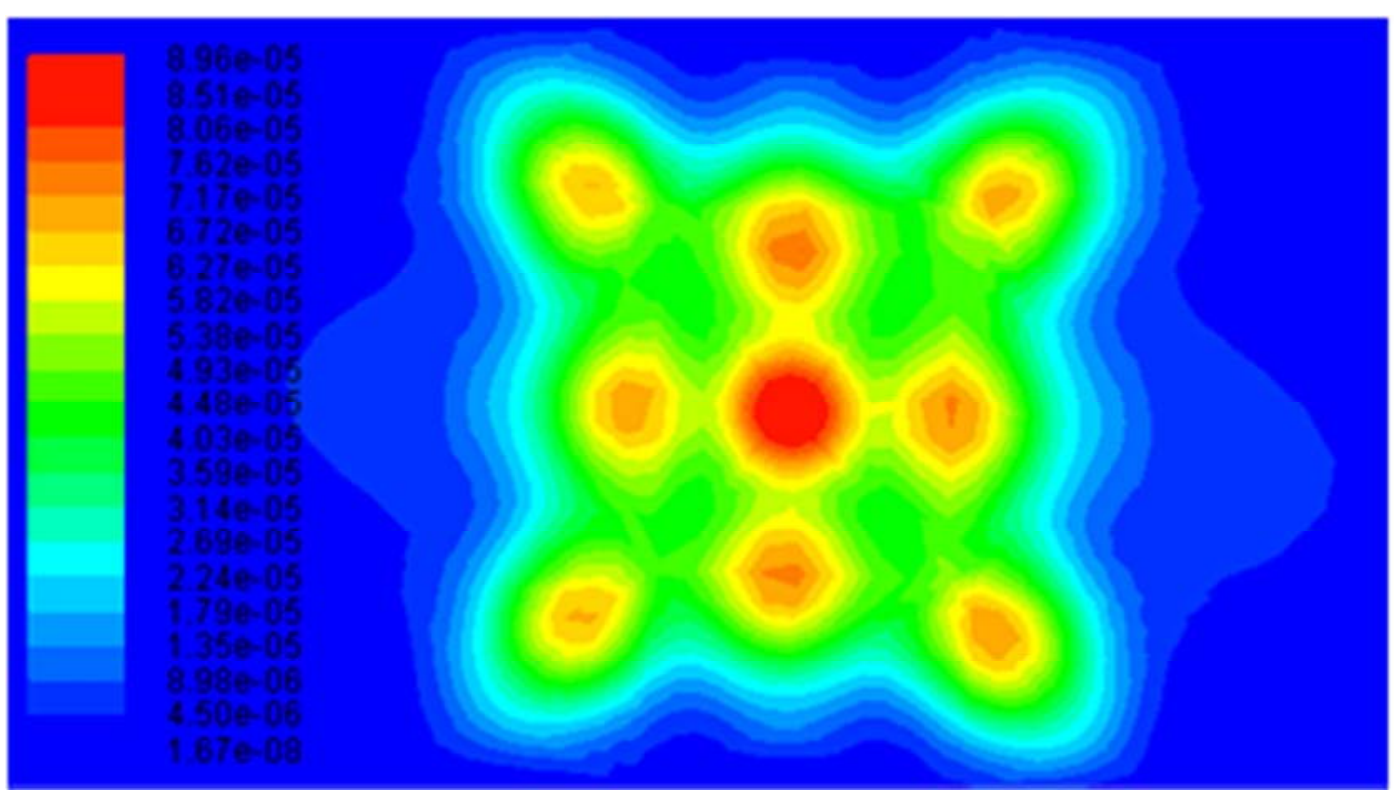

Figure 6. The concentration of welding fume in cross section of $Z=1 \mathrm{~m}$ and $1.5 \mathrm{~m}$ spacing Keep $X, Y$-axis unchangeable, $Z=1 \mathrm{~m}$, under the no-wind condition, the situation of welding fume diffusion is shown in Figure 7. 


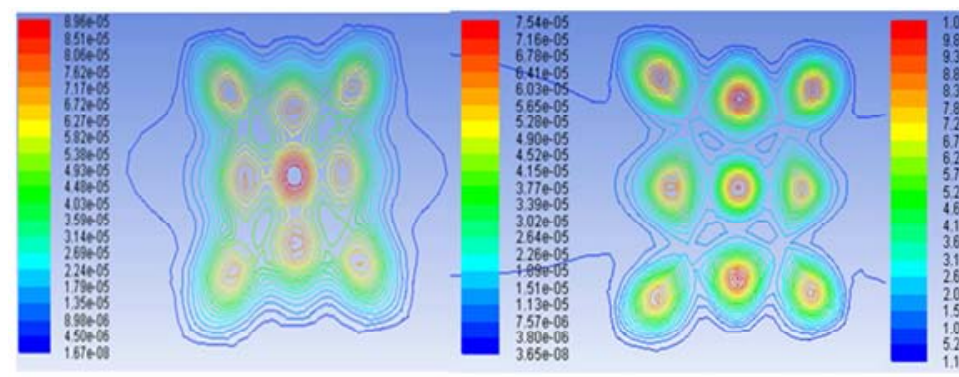

(a) $1.5 \mathrm{mspacing}$ (b) $1.8 \mathrm{mspacing}$

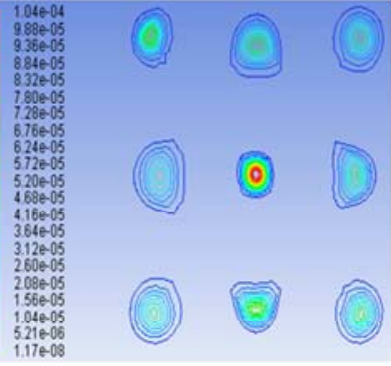

(c) 2 mspacing

Figure 7. The isoline map of concentration distribution in different distance under $Z=1 \mathrm{~m}$.

As can be seen from Figure 7 that in same cross-section, the interaction between dust sources decreases as the distance between the dust sources increases. At the same time, when in different cross-section, the influence of variant dust sources on the decline of welding fume concentration is shown in Figure 8.

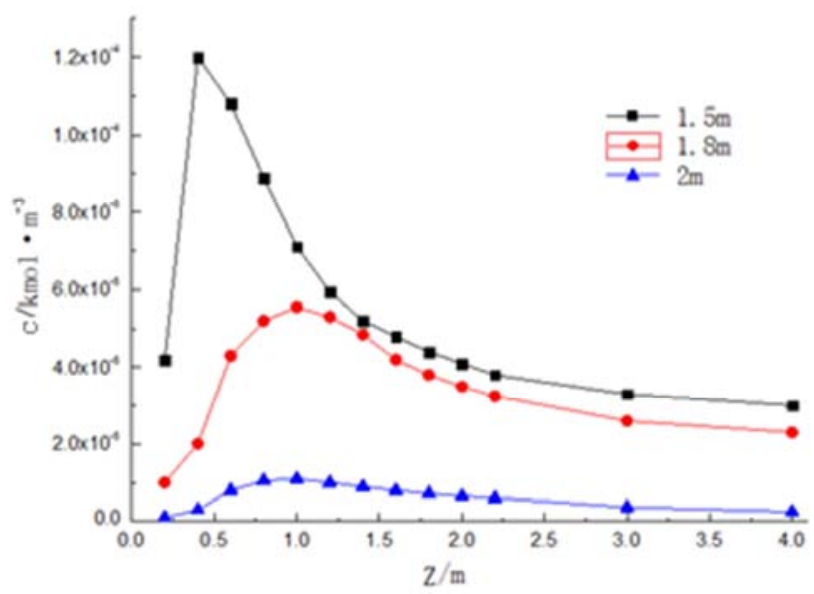

Figure 8. The isoline map of concentration distribution in different distance under $Z=1 m$.

From Figure 8, the following conclusions can be drawn:

(1) When the distance is same, the different height in vertical direction of the dust sources leads to a process that the welding fume dust concentration increase rapidly first and then decrease smoothly. While the height is less than $1 \mathrm{~m}$, the change process of dust concentration is faster, and if the height raised to $2 \mathrm{~m}$, the change process of dust concentration tends to be gentle.

(2) When the cross-section is same, the larger distance between the dust sources causes a lower concentration and a faster concentration decreasing speed of the dust. The dust concentration at $1.5 \mathrm{~m}$ distance is much higher than that of the $2 \mathrm{~m}$ distance. While $\mathrm{Z}=0.5 \mathrm{~m}$, the dust concentration reach the highest point when the distance of dust sources is $1.5 \mathrm{~m}$, and only when the vertical height of dust source is $1 \mathrm{~m}$, the dust concentration reach the highest point when the distance is $1.8 \mathrm{~m}$ or $2 \mathrm{~m}$.

\subsubsection{The Influence of Airflow on Welding Fume}

In order to better show the simulation results, the $3 \mathrm{~m}$ distance between the dust source was chosen, and simulating the diffusion at different wind speeds.

Keep the X, Y-axis unchangeable, While $\mathrm{Z}=0.5 \mathrm{~m}$, Set the situation that 9 dust sources exist at the same time under no wind conditions, and the distance between the dust sources is $3 \mathrm{~m}$, The concentration distribution of the welding fume is shown in Figure 9.

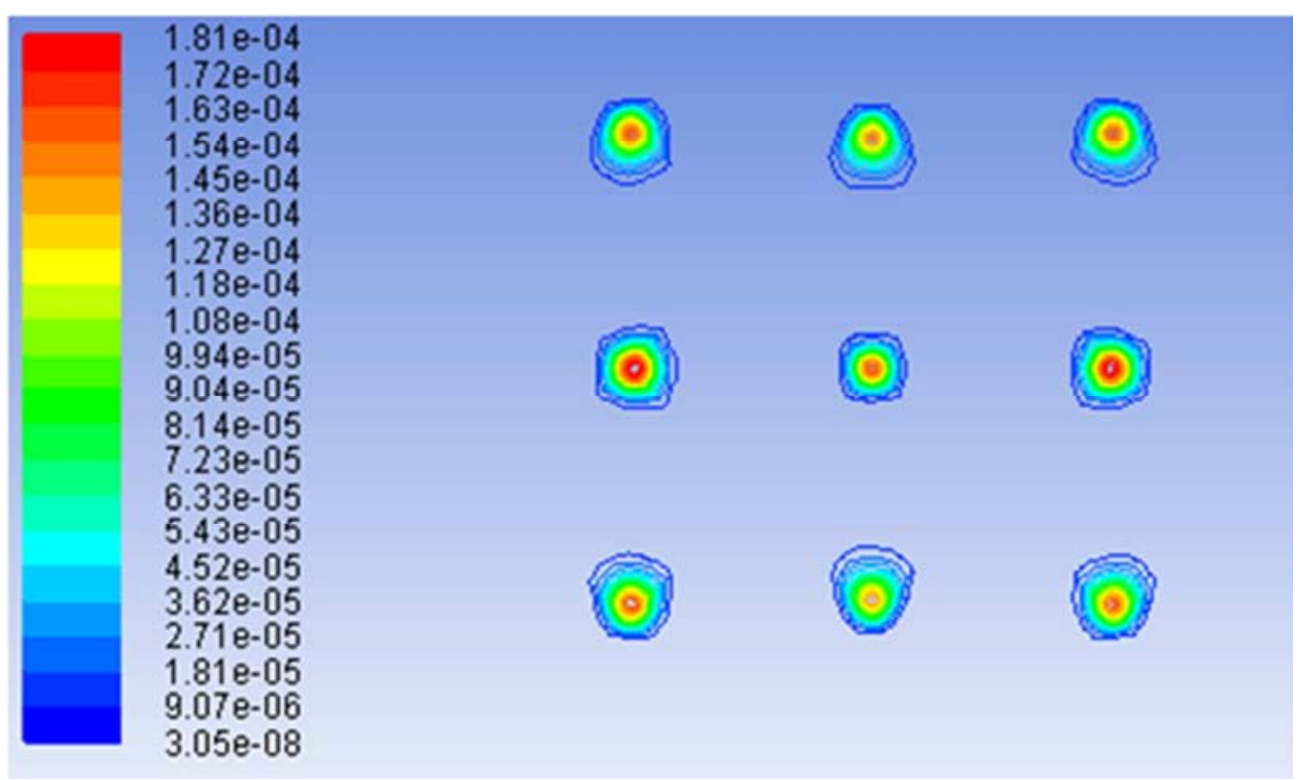

Figure 9. The contour of welding fume concentration distribution of the $Z=0.5 \mathrm{~m}$ section of the map under no wind contidions. 
When the dust source is 9 , under the condition of the $3 \mathrm{~m}$ distance between the dust source, simulating the diffusion of welding fume when the wind speed is $0.8 \mathrm{~m} / \mathrm{s}, 1 \mathrm{~m} / \mathrm{s}, 1.2 \mathrm{~m} / \mathrm{s}$.

Meanwhile, the two doors on one side and the middle of the four windows are set to speed inlet, the other side of the door and the middle of the four windows for the pressure outlet, the simulation time is chosen for 10 minutes.

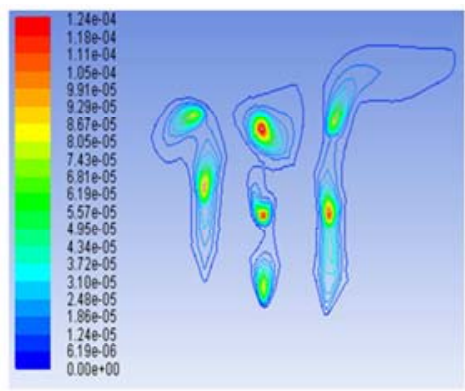

(a)Wind speed of $0.8 \mathrm{~m} / \mathrm{s}$

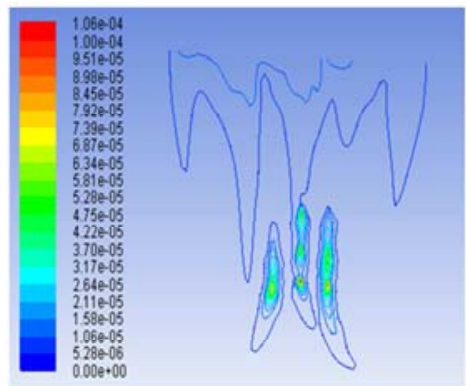

(b) wind speed of $1 \mathrm{~m} / \mathrm{s}$

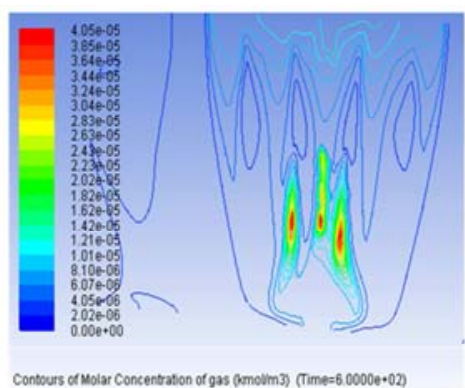

(c) wind speed of $1.2 \mathrm{~m} / \mathrm{s}$

Figure 10. The isoline map of the welding fume concentration distribution in $z=0.5 m$ section.

From the diagram of the simulation results can be seen, in no wind condition, dust source distance closer, smoke concentration is higher; under different wind speed and other conditions are same, the wind speed is high, the dust concentration is smaller, faster diffusion.

Of course, the position of the exhaust hood all have a great impact on reducing the concentration of welding fume. From the simulation, as the distance declines, the time that welding fume reaches the smoke exhausting machines declines and the speed of the welding fume increases.

\section{Control Technology}

As can be seen from the above simulation, the vertical direction is the main direction of dust diffusion, and finally the whole workshop would be filled with fume dust. There are many factors that influence the diffusion, such as the speed of wind, the distance between welding operating positions, the vertical distance of welding operation and the designs of exhaust hood. In this paper, the rotary air curtain exhaust hood is used to capture the welding fume.

The rotary air curtain exhaust hood is a kind of device that uses artificial tornado to exhaust dust in the workshop. Through the outer layer of the exhaust hood, the fresh air is delivered outside, which would generate a jet-flow to isolate the dust source and the outside air. Then, due to the function of the inner layer of the exhaust hood, a circulating airflow and a negative pressure center are generated in the dust flow, and under the interaction of the circulating airflow and the negative pressure center, avortex is generated in the dust flow, which could promote the welding fumes to be inhaled by the suction port $[9,15]$

In the workshop, exhaust hoods are installed $2 \mathrm{~m}$ above of each operation point, each exhaust hood generates an artificial tornado in vertical vortex structure under the interaction between the circulating airflow and the inhaling process of the suction entrance, and a stable cyclone would be formed in the cross section. Such a tornado leads to a negative pressure center near the lower axis under the hood, the air flow is converged in the center under the hood, and with the interaction between the airflow and inhaling air flow, pollutants are exhausted and the efficacy of inhaling entrance is promoted effectively. A noticeable aspect of this process is that, the fresh cool air that is ejected from jet-flow exit could reduce the temperature of workshop effectively, which would keep the welding workers in a comfortable working condition lest the high-temperature working condition is detrimental to their health [16]. The flow field diagram is shown in Figure 11.

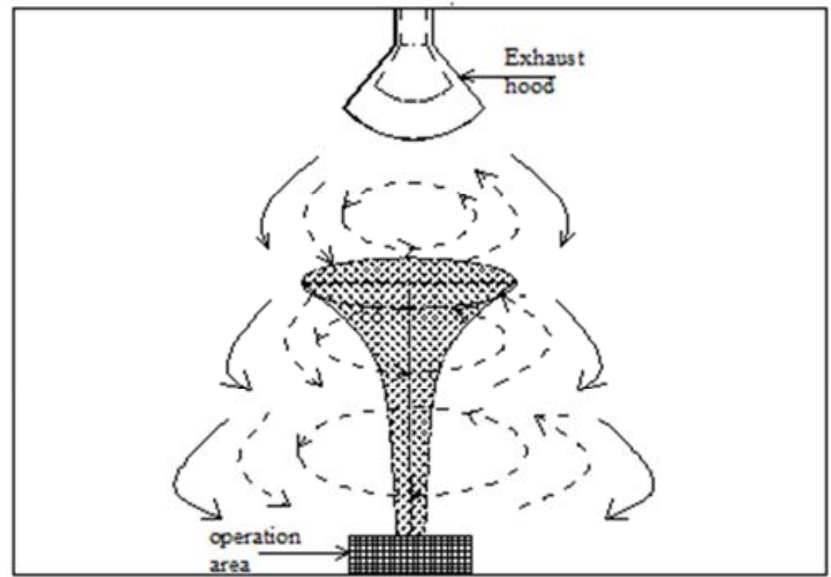

Figure 11. The flow field diagram of the rotary air curtain exhaust hood.

As can be seen from Figure 11, in dust rising process of the welding, the suction effect of the exhaust hood suction port leads to a negative pressure in welding dust, at the same time, a vortex is formed, which drives the welding dust rising continuously. In the welding dust rising process, the condensing phenomenon might happen among the tiny particles in the dust, but if the speed of the suction outlet is fast enough, the progress of condense would be delayed. According to Shi Yuxiang's research, the particles with the size of $10^{-2}$ umare mainly generated though homogeneous nucleation of vapor to particles, and such particles is usually the kind of particle that human body absorbs. In the process of Fig. 9, when the dust particles are still steam, the dust particles have been inhaled as the 
formation of secondary particles by the exhaust hood already, which could collect the harmful substances generated during welding operation effectively [17].

\section{Conclusions}

(1) In the single welding operation, under theno-wind condition, keep X, Y-axis unchangeable, the dust distribute evenly in the vertical direction, and the dust diffusion become faster when $\mathrm{Z}$ increase, the simulation above shows that, when $\mathrm{Z}$ is bigger than $1 \mathrm{~m}$, the concentration of dust diffusion is smaller and the trend is smoother, when keeping the $\mathrm{X}, \mathrm{Z}$-axis unchangeable, the dust would spread to the surrounding in horizontal direction, and the concentration decreases as the distance becomes larger.

(2) In the dense welding operation, the larger distance between the dust sources causes a lower concentration of the dust and a smaller interaction between the dust sources, and the faster wind speed leads to a lower concentration of dust, in the cross-section which is closer to the ground, the airflow speed have more apparent influence on the dust concentration, which would decrease the concentration of dust rapidly. When the distance between the dust sources and the fume exhaust machine also has influence on it, as the distance declines, the time that welding fume reaches the smoke exhausting machines declines and the speed of the welding fume increases.

(3) The rotary air curtain exhaust hood is used to collect the welding fume, which can not only absorb the toxic and harmful gases produced in welding operation, but also improve the comfort feelings of welders in the operation, and inhaling welding dust absorbed by workers decreases, the incidence of welder-pneumoconiosis would be reduced effectively.

\section{References}

[1] Bin, P. F., Zhang, M. B, Chen, J. et al. 2012. A study on exposure characteristics of submicron particles and fine particles in welding workplace. Zhejian Preventive Medicine, 24(2):8-12.

[2] Li, S. 2014. Numerical simulation of the electrohydraulic field in electrostatic welding smoke purifier. Donghua University.

[3] Marconi M, Bravaccini A. 2010. Capture efficiency of integral fume extraction torches for gma welding-part 1 . Welding in the world, 54(1):3-15.
[4] Cai, Z. P., Xu, Z. G. and Shi, J. K. 2003. Discussion on the diffusion model of welding fume in large ship welding workshop. Journal of Donghua University, 29(2):27-31.

[5] Niu, P. P. and Zhao, J. P. 2013. Research on the dispersion regularity of electric welding workshop air pollutant under the condition of ventilation control. Xi'an University Of Architecture And Technology.

[6] Liu, Z. Y., Jia, Y. Y. and Zhang, Y. J. 2014. Experimental study on the distribution of welding fume concentration. Science Technology-and Engineering, 17(14):178-181.

[7] Ministry of health of the people's Republic of China GB/T 2.1-2007 workplace hazardous factors occupational exposure limit chemical hazardous factors. China Standards Press, 2007 (in Chinese).

[8] Dong, L. G. and Lin, B. 2003. Research of suction-airflow affected by annular jet. Ventilating and Air Conditioning, 33(3):27-29.

[9] Wang, P. F. 2009. Numerical Simulation and Experimental Research of the Rotary Air Curtain Exhaust Hood. Hunan University of Science and Technology.

[10] Yang, Y., Wang, Y. and Li, Y. B. 2015. Flow field characteristics of exhaust flow assisted with a jet. Journal of Southwest Jiao Tong University, 50(2):347-351.

[11] Yu, Y. 2008. FLUENT introductory and advanced tutorial. Beijing Institute of Technology press.

[12] Wang, L. He, Y. Tang, M. K, and et al. 2016. CFD simulation of gas diffusion. Jiangxi University of Science and Technology, 37(3):13-18.

[13] Pang, Y. J., Chen, J. J. and Fu, S. H. 2014. Numerical simulation of air distribution of up-supply and down-return in laboratory. Building Energy Efficiency, 279(42):1-4.

[14] Xia, X. B. 2015. Experimental study on air quality of displacement ventilation welding workshop. ShanXi Archtiecture, 41(13):123-125.

[15] Wang, Z. W. and Guo, C. 2010. Study on the coupling mechanism of the new displacement ventilation system. Fluid Machinery, 38(6):1-10.

[16] Pan, Y. Wang, P. F. and Wang, W. J. 2011. Experimental study on the new type of rotary air curtain exhaust hood. Refrigeration and Air Conditioning, 25(6):562-565.

[17] Shi, Y. X., Xiang, S. X. and Yang, S. B. 1997. A study on homogeneous nucleation mechanism of welding aerosol particles. Journal of Wuhan University of Technology, 21(4):396-400. 